\title{
A tyrosine PEG-micelle magnetic resonance contrast agent for the detection of lipid rich areas in atherosclerotic plaque
}

\author{
Anne Beilvert ${ }^{1,2}$, David P. Cormode ${ }^{2}$, Frédéric Chaubet ${ }^{1}$, Karen C. Briley-Saebo ${ }^{2}$, Venkatesh \\ Mani $^{2}$, Willem J.M. Mulder ${ }^{2}$, Esad Vucic ${ }^{2}$, Jean-François Toussaint ${ }^{3}$, Didier Letourneur ${ }^{1}$, and \\ Zahi A. Fayad ${ }^{2}$ \\ Anne Beilvert: ; David P. Cormode: ; Frédéric Chaubet: ; Karen C. Briley-Saebo: ; Venkatesh Mani: ; Willem J.M. Mulder: ; \\ Esad Vucic: ; Jean-François Toussaint: ; Didier Letourneur: ; Zahi A. Fayad: Zahi.Fayad@mssm.edu \\ 1 Inserm U698, Cardiovascular Bio-engineering, CHU X. Bichat, University Paris 7, Paris, F-75877; \\ Institut Galilée , University Paris 13, Villetaneuse F-93430, France \\ 2 Translational and Molecular Imaging Institute, Mount Sinai School of Medicine, One Gustave L. \\ Levy Place, Box 1234, New York, NY 10029, Tel: 212-241-6858, Fax: 240-368-8096 \\ 3 Université Paris Descartes and Inserm U652, Pôle Imagerie et Explorations Fonctionnelles, Hôtel- \\ Dieu, 75181 Paris Cedex 4, France
}

\section{Abstract}

Vulnerable or high-risk atherosclerotic plaques often exhibit large lipid cores and thin fibrous caps that can lead to deadly vascular events via their rupture. In this study, PEG-micelles that incorporate a Gd-DTPA amphiphile were used as an MR contrast agent. In an approach inspired by lipoproteins, the micelles were functionalized with tyrosine residues, an aromatic, lipophilic amino acid, to reach the lipid rich areas of atherosclerotic plaque in a highly efficient manner.

These micelles were applied to apolipoprotein E -/- mice as a model of atherosclerosis. The abdominal aortas of the animals were imaged using $\mathrm{T}_{1}$-weighted high-resolution MRI at $9.4 \mathrm{~T}$ before and up to 48 hours after the administration of the micelles. PEG-micelles modified with $15 \%$ tyrosine residues, yielded a significant enhancement of the abdominal aortic wall at 6 and 24 hours postinjection as compared to unmodified micelles. Fluorescence microscopy on histological sections of the abdominal aorta showed a correlation between lipid rich areas and the distribution of the functionalized contrast agent in plaque.

Using a simple approach, we demonstrated that lipid-rich areas in atherosclerotic plaque of ApoE -l - mice can be detected by MRI using Gd-DTPA micelles.

\section{Keywords}

atherosclerosis; contrast agent; MRI; lipid core; micelle

\section{Introduction}

The consequences of atherosclerosis represent a critical public health concern in western countries and are likely to become the major public health problem in the developing world in the near future (1). One of the key initial steps in atherogenesis is the subendothelial retention of apolipoprotein B-containing lipoproteins (2). Plaque formation eventually leads to luminal

Corresponding author : Zahi A. Fayad Translational and Molecular Imaging Institute, Mount Sinai School of Medicine, One Gustave L. Levy Place, Box 1234, New York, NY 10029, Tel: 212-241-6858, Fax: 240-368-8096, Zahi.Fayad@mssm.edu. 
narrowing which can be detected by magnetic resonance imaging (MRI). Early detection of atherosclerotic plaque, however, is challenging but could aid in the prevention of acute cardiovascular events such as stroke or heart attack. Another important application could be the assessment of the efficacy of treatment strategies targeted at atherosclerotic plaque stabilization or regression. The use of magnetic resonance contrast agents improves the detection of morphological and functional abnormalities within the vessel wall (3-6). Gadolinium (Gd) based contrast agents are commonly used in MRI to improve contrast between tissues (7) and several contrast agents targeting the atherosclerotic plaque are currently under investigation (8-10). They have been designed to detect different cellular and molecular plaque features, including macrophages, neovessels, activated endothelium or thrombus in advanced lesions. This variety is due to the highly complex progression of atherosclerosis, which results in very heterogeneous plaques with highly fibrous structures, calcifications, inflamed tissue, lipid pools and combinations of the above (11).

In the current study, we aimed to image the lipoproteins of lipid deposits in the plaque by conceptually basing our agent on the human apolipoprotein A-I mimetic peptide D-4F (12). D4-F is an amphiphatic peptide with both a polar and a nonpolar face. This structure allows it to mimic the lipid-binding properties of apolipoprotein A-I (12-14). The lipophilic side of D-4F contains 4 hydrophobic phenylalanine residues (15). We chose tyrosine as a simple hydrophobic moiety. The tyrosine groups are also easily quantifiable by UV spectroscopy for chemical characterization. Therefore, we hypothesized that functionalized structures, such as nanoparticles, exposing multiple tyrosine groups at their surface could be used to identify lipidrich areas in atherosclerotic plaques. Previously reported targeted contrast agents have used antibodies (16), antibody fragments, proteins (17), peptides, peptidomimetics (18) and small molecules (19) for targeting purposes. Using only tyrosine residues on the surface of the contrast agent is a simple approach in comparison.

The MR contrast agent reported here is based on a platform we introduced recently, i.e. PEGmicelles that include Gd-DTPA based lipids (20). This platform has been used to image macrophages in plaque and oxidized LDL and has a greater half-life than regular micelles $(21,22)$ due to the protective "stealth" effect of the outer shell of PEG chains, which reduces recognition by opsonins and consequent clearance $(22,23)$.

The aim of the current study was to prepare, characterize and evaluate in vivo the efficacy of tyrosine micelles using an apolipoprotein E knock-out (ApoE-/-) mouse model on a high cholesterol, Western-type diet (24). MRI of the abdominal aorta was performed on a $9.4 \mathrm{~T}$ system, before and at several time points after injection of micelles. As controls, the tyrosine micelles were injected in wild-type mice, while non-derivatized micelles were applied to ApoE $-1-$ mice as well.

\section{Methods}

\section{Materials}

1,2-Distearoyl-sn-glycero-3-phosphoethanolamine-N-[carboxy(polyethylene glycol)2000] (ammonium salt) (carboxy-PEG 2000 -DSPE), 1,2-distearoyl-sn-glycero-3phosphoethanolamine-N-[methoxy(polyethylene glycol)-2000] (ammonium salt) ( $\mathrm{PEG}_{2000^{-}}$ DSPE) and 1,2-dipalmitoyl-sn-glycero-3-phosphoethanolamine-N-(lissamine rhodamine B sulfonyl) (ammonium salt) (Rhodamine PE) were purchased from Avanti Polar Lipids (Alabaster, AL, USA). L-tyrosine methylester hydrochloride, 1-ethyl-3-(3dimethylaminopropyl) carbodiimide hydrochloride (EDC), N-hydroxysuccinimide (NHS), pyrene, sodium hydroxide $(\mathrm{NaOH})$ and chlorhydric acid $(\mathrm{HCl})$ were purchased from Sigma (St Louis, MO, USA). Gd-DTPA-bis-stearylamide (Gd-DTPA-BSA) was from iQSynthesis (St Louis, MO, USA). 
For fluorescent microscopy, monoclonal anti-mouse CD68 conjugated AlexaFluor® 488 was purchased from Serotec (Raleigh, NC, USA). Rabbit anti-mouse collagen I polyclonal antibody was from Millipore (Billerica, MA, USA), rabbit polyclonal $\alpha$-smooth muscle actin and rabbit polyclonal anti-apolipoprotein B whole serum were from Abcam (Cambridge, UK) and goat anti-mouse decorin monoclonal antibody was from R\&D Systems (Minneapolis, MN, USA). Secondary antibody goat anti-rabbit AlexaFluor ${ }^{\circledR} 488$ and donkey anti-goat AlexaFluor® 488 were both from Molecular Probes, Invitrogen (Carlsbad, CA, USA). Nuclei were stained with DAPI (Invitrogen, Carlsbad, CA, USA).

\section{Animals}

The Institutional Animal Care and Use Committee of Mount Sinai School of Medicine approved animal procedures. All mice were purchased from Jackson Laboratories (Bar Harbor, ME, USA). ApoE- $-1-$ mice $(n=22)$ with a mean age of $11 \pm 2.6$ month-old male were used for this study. Age matched wild-type (WT) were used as a negative control $(\mathrm{n}=3)$. The ApoE $-/-$ mice were fed a Western Diet for at least 24 weeks (mean 36,5 \pm 11 weeks) before the experiment (WD, containing 21\% fat, $0.15 \%$ cholesterol; Diet D01022601 Research Diets Inc (New Brunswick, NJ, USA)).

\section{Micelle preparation}

Micelles were prepared by mixing $0.05 \mathrm{mmol} \mathrm{Gd}$-DTPA-bis-stearylamide, $0.018 \mathrm{mmol}$ $\mathrm{PEG}_{2000}$-DSPE and $0.032 \mathrm{mmol}$ carboxy-PEG-2000-DSPE in a round bottom flask. The lipids were dissolved in chloroform/methanol 4:1 (v/v) and 0.1\% Rhodamine-PE was added as a fluorescent label. A thin film was obtained by slowly removing the solvents under vacuum at $65^{\circ} \mathrm{C}$. The film was hydrated by adding $4 \mathrm{ml}$ warm PBS buffer $\left(70^{\circ} \mathrm{C}\right)$ to the film, which was maintained at the same temperature. The solution was stirred at $70^{\circ} \mathrm{C}$ until a clear solution was formed.

Tyrosine residues were coupled to the surface of the micelles, as shown in Figure 1. L-tyrosine methylester was coupled to the carboxyl groups on the carboxy-PEG lipids by adding 0,046 mmol EDC, 0,1 mmol NHS and 0,03 mmol L-tyrosine methylester to the micelle solution. The micelle solution was cooled to $4^{\circ} \mathrm{C}$ and the $\mathrm{pH}$ decreased to 4.2 by the addition of diluted $\mathrm{HCl}$. The EDC was added and the $\mathrm{pH}$ increased to 6.8 (via $\mathrm{NaOH}$ addition). The NHS and amino acid was then added and the $\mathrm{pH}$ raised to 10 (via $\mathrm{NaOH}$ addition). The sample was mixed for 30 minutes until the reaction was stopped by decreasing the $\mathrm{pH}$ to 7 (via addition of $\mathrm{HCl}$ ). Purification was achieved by washing the functionalized micelles with PBS 3 times (centrifugations were performed using Vivaspin ${ }^{\circledR}$ tubes (Sartorius Corporation, Edgewood, NY, USA) cut-off $10 \mathrm{kDa}$ ), at $4^{\circ} \mathrm{C}$, for $20 \mathrm{~min}$, at $4000 \mathrm{rpm}$ ).

Unmodified micelles were made as described above. They were composed of 50\% Gd-DTPABSA, 50\% PEG-2000-DSPE and $0.1 \%$ rhodamine-PE. These $\%$ values are based on moles of lipids.

\section{Characterization}

The size of the micelles was determined by dynamic light scattering using a Malvern Instruments HPPS device (Malvern Instruments, Malvern, UK). The mean diameter at $25^{\circ} \mathrm{C}$ was determined based upon both volume and number weighting. Relaxivity and gadolinium (Gd) content were measured using a Minispec mq60 NMR Analyser (Bruker, Karlsruhe, Germany), as described previously (25).

Micelle stability over time was assessed by studying the evolution of particle size distribution by dynamic light scattering over time. Critical micelle concentrations (CMC) were determined in serum or PBS as a measure of micelle stability, using pyrene as a fluorescent probe as 
previously described (26). Briefly, aliquots of pyrene solutions in acetone were added to vials, and acetone was allowed to evaporate. $1 \mathrm{~mL}$ of tyrosine micelles at different concentrations in PBS/serum was then added to the vials giving a final concentration of $6 \times 10^{-7} \mathrm{M}$ of pyrene. The solution was incubated overnight at $50^{\circ} \mathrm{C}$ to reach pyrene equilibrium. Fluorescence spectra was recorded on a F-2000 fluorescence spectrophotometer (Hitachi) using $\lambda_{\mathrm{ex}}=339$ $\mathrm{nm}$ and $\lambda_{\mathrm{em}}=350-450 \mathrm{~nm}$. Fluorescence intensity at $375 \mathrm{~nm}$ was plotted as a function of tyrosine micelle concentration.

The L-tyrosine methylester content was assayed by UV spectrometry at $280 \mathrm{~nm}$ from the second order derivative of the spectra (UVmc2, Safas, Monaco) and by comparison to a calibration curve.

The blood half-life of tyrosine micelle was determined in ApoE-/- mice $(\mathrm{n}=6)$ as previously described for unmodified micelles $(22,27)$. After tyrosine micelle injection blood was drawn into heparinized blood collections tubes via heart puncture at different time points postinjection, from $5 \mathrm{~min}$ to $24 \mathrm{hrs}$ post-injection.

\section{MR imaging}

The abdominal aorta was scanned using a 9.4 T MR imager (Bruker, Karlsruhe, Germany) equipped with a $30 \mathrm{~mm}$ transmit/receive birdcage coil. Mice were anesthetized continuously with a mixture of oxygen and isoflurane $(0.5-2 \%)$. The contrast agents were administered to the animals $(60 \mu \mathrm{mol} \mathrm{Gd} \mathrm{per} \mathrm{kg})$ via injection into the tail vein. Imaging was performed preand $6 \mathrm{hrs}, 24 \mathrm{hrs}$, and $48 \mathrm{hrs}$ post-injection (pi).

Mice were scanned using the following protocol. The abdominal aorta was identified in a coronal section on a localizing sequence. T1-weighted MRI was performed using a black blood turbo spin echo sequence with arterial saturation bands. Sixteen contiguous $500 \mu \mathrm{m}$-thick slices with a microscale in-plane resolution of $101 \mu \mathrm{m}$ were acquired using a spin echo sequence with a $256 \times 256$ matrix size. The repetition time (TR) and echo time (TE) for the T1W images were 800 and $8.6 \mathrm{~ms}$, respectively. An inflow saturation band of $3 \mathrm{~mm}$ was used with a slice gap of $3 \mathrm{~mm}$ for additional arterial luminal flow suppression. Sixteen signal averages were used for a total imaging time of $55 \mathrm{~min}$ per scan. A saturation pulse was used to eliminate signal from fat tissue and better delineate boundary of the aortic wall and minimize chemical shift artifacts.

At each time point the slices were matched to the baseline pre-contrast scan by using the unique vertebral and paraspinous muscular anatomy as landmarks. Five slices per study were chosen that were of good image quality, without or with little flow or motion artifacts at the 4 time points. The signal intensity of the atherosclerotic plaque and the paraspinous muscle were measured using eFilm Workstation software (Merge Technologies Inc. Milwaukee, WI, USA). Since the plaques of mice are particularly heterogeneous (28), only the enhancing areas of the aortic wall were used to quantify the signal. The standard deviation (SD) of noise from the background was also obtained.

For each slice the percent enhancement (\% ENH) (25), relative to the muscle, was calculated (according to the equation below) in order to evaluate the enhancement of the aortic wall.

$\% \mathrm{ENH}=(\mathrm{CNRpost} / \mathrm{CNRpre}-1) \times 100$ with $\mathrm{CNR}=\mathrm{SIwall} / \mathrm{SImuscle}$, where SI is the signal measured in the tissue.

As controls, a group of WT mice $(n=3)$ was injected with tyrosine micelles and imaged at the same time points. Two groups of ApoE- $-1-$ mice were injected with unmodified micelles and imaged either at 6, 24 and 48 hours $(n=5)$ or at earlier time point 30 min and 2 hours $(n=3)$.

Magn Reson Med. Author manuscript; available in PMC 2010 November 1. 


\section{Fluorescence microscopy}

Conventional fluorescence microscopy was performed on sections of abdominal aortas of ApoE - /- mice that matched the areas of enhancement in MR images from ApoE-I-mice. Mouse aortas were removed 6 hours after the micelle injection, embedded in TissueTek® (Sakura, Torrance, CA, USA), immediately placed on dry ice and kept at $-80^{\circ} \mathrm{C}$ until sections were obtained. $7 \mu \mathrm{m}$ frozen sections were fixed in PBS buffered $4 \%$ paraformaldehyde solution for $15 \mathrm{~min}$. After fixation, sections were rinsed two times with PBS $1 \mathrm{X}$ (15 min each). Then, sections were blocked with PBS containing 5\% goat serum or BSA for $30 \mathrm{~min}$ at room temperature for further staining.

After blocking, sections were incubated with one primary antibody against CD68 macrophages dilution 1/20, decorin (1/15), collagen I (1/40), apolipoprotein B (ApoB) (1/50) or $\alpha$-smooth muscle actin (1/50) in the blocking solution for 2 hours at room temperature. After washing, the sections were stained with a secondary antibody tagged with a fluorophore at RT for 1 hour, except in the case of the macrophage staining; that primary antibody was fluorescently tagged. They were both diluted to $1 / 500$. Sections were washed then stained for nuclei with DAPI and finally were mounted with Dako fluorescent mounting medium. Finally several types of control experiments were performed for fluorescence microscopy. Matched ApoE-/- mice were sacrificed and stained with secondary antibodies alone. The sections demonstrated no significant fluorescence (data not shown). Controls were also performed to confirm that the red fluorescence obtained from aortic sections after tyrosine grafted micelles injection is unrelated to autofluorescence.

Fluorescence microscopy was performed using a Nikon Eclipse E400 (Nikon, Japan). Pictures were analyzed with Archimed 5.2.2 software (Microvision, Evry, France). Gain and exposure time were independently determined for each set of antibodies.

\section{Statistical analysis}

Statistical analysis was performed using SPSS 14.0 for Windows. A paired t-test analysis and a one way Analysis of Variance (ANOVA) with Bonferroni's multiple comparison test were performed to determine the significance of wall enhancement of the experimental groups as compared to the control group. P-values less than 0.05 were considered significant.

\section{Results}

\section{Tyrosine grafted micelles}

Dynamic light scattering revealed that the micelles (Figure 1) composed of 50\% PEG-lipids and 50\% lipids bearing Gd-DTPA had a mean hydrodynamic diameter below $20 \mathrm{~nm}$. Micelles that had tyrosine conjugated to the surface did not significantly differ in size and relaxivity from the unmodified micelles (Table 1). Unmodified and tyrosine micelles showed a single peak $(>99 \%)$ on number-weighted chromatograms, indicative for a narrow size distribution. The values found for the unmodified and tyrosine micelle sizes were $12 \pm 2.7 \mathrm{~nm}$ and $14.6 \pm$ $2.5 \mathrm{~nm}$, respectively. The longitudinal relaxivity $\left(\mathrm{r}_{1}\right)$ was not significantly affected by grafting of tyrosine on the PEG-chains as $r_{1}=10.1 \pm 1.5 \mathrm{mM}^{-1} \mathrm{~s}^{-1}$ for unmodified micelles while $\mathrm{r}_{1}=$ $10.3 \pm 0.9 \mathrm{mM}^{-1} \mathrm{~s}^{-1}$ for tyrosine micelles. Moreover, both micelles were found to be stable for over a month at room temperature via negligible changes in size and relaxivity.

Unmodified micelles have been described $(23,29)$ with a very low critical micelle concentration with a value of about $10^{-5} \mathrm{M}$. Tyrosine micelles had a CMC both in PBS and in serum of $2.5 \times 10^{-6} \mathrm{M}$. These in vitro experiments indicate that both types of micelles will maintain their integrity even at high dilution. In comparison, the initial concentration of micelles in the blood of mice after injection is $1.8 \times 10^{-3} \mathrm{M}$, i.e. 3 orders of magnitude greater than the CMC. 105 
$\mu \mathrm{L}$ of $34 \times 10^{-3} \mathrm{M}$ micelle were injected in mouse which have a blood volume around $2 \mathrm{~mL}$. Tyrosine conjugation yield was established to be $25 \%$, resulting in $15 \pm 4 \%$ of the PEG chains conjugated with tyrosine.

After injection of micelles in ApoE-/- mice, we found that adding tyrosine to the micelles increased the blood half-life from 2 hours for the unmodified micelles to 21 hours for tyrosine micelles (data not shown).

\section{Enhancement of tyrosine grafted micelles}

The micelles were intravenously applied to an ApoE-I- mouse model of atherosclerosis that had been on a high fat and high cholesterol diet, and to wild-type mice on a normal diet to evaluate the efficacy of these contrast agents for early atherosclerosis imaging. Mice were injected with tyrosine micelles or unmodified micelles at a dose of $60 \mu \mathrm{mol} \mathrm{Gd} / \mathrm{kg}$ via the tail vein. Before injection, all mice underwent a baseline scan for comparison, which was followed by scans at 6,24 and 48 hours post-injection (pi).

A group of ApoE-I- mice $(\mathrm{n}=8)$ was injected with tyrosine grafted micelles. The administration of tyrosine micelles in the ApoE-/- mice resulted in a significant increase in the vessel wall MRI signal (Figure 2) at 6 hours and 24 hours pi. At 48 hours pi, very little enhancement could be observed indicating that the agent had left the plaque at this time point and that repeat imaging would be possible with this agent in this relatively short timeframe. The signal coming from the vein wall is probably due to circulating contrast agent or to blood flow artifact, since blood flow saturation bands were only used on the arterial side.

The mean wall enhancement due to tyrosine grafted micelles in the aorta wall of ApoE-/mice and unmodified micelle in ApoE- $-(\mathrm{n}=5)$ was determined at 6, 24 and 48 hours pi (Figure 3). This wall enhancement with the tyrosine grafted micelles was very high at 6 hours $(104 \% \pm 58)$ and remained elevated until 24 hours $(61 \% \pm 41)$ post injection. Thereafter the enhancement decreased and the vessel wall MR signal intensity returned to baseline after 48 hours pi. Analysis of the data obtained from the injection of unmodified micelles in ApoE-/mice did not show any significant enhancement at 6 or 24 hours, with the mean values for these time points being $35 \pm 13 \% \mathrm{ENH}$ and $13 \pm 17 \% \mathrm{ENH}$, respectively (Figure 3). Statistical testing performed on the unmodified micelles data showed no significant enhancement at any time point.

At 6 hours and 24 hours, the enhancement obtained with tyrosine grafted micelles was significantly different from the enhancement from the unmodified micelles in the ApoE-/ - group ( $<<0.05$ ANOVA).

As additional controls, a group of WT mice $(\mathrm{n}=3)$ was imaged before and after the injection of tyrosine micelles at the same time point (6, 24 and 48 hours pi). There was no apparent enhancement at any time point, the \% ENH being not significantly different from background signal.

CNR values were also measured before the injection, 6, 24 and 48 hours after the injection. Pre-injection CNR values were not different among the different groups of mice $(\mathrm{p}>0.05)$ respectively for unmodified micelles in wild-type mice $1.03 \pm 0.33$, in ApoE $-/-$ mice $0.99 \pm$ 0.25 and for tyrosine micelle in ApoE-/- mice $0.8 \pm 0.23$. In ApoE-/- mice injected with tyrosine micelle, the CNR at the 6 hours post-injection time-point $(1.60 \pm 0.45)$ was significantly different from the CNR in the pre-injection images $(0.80 \pm 0.2)$, confirming that the signal increased in the aortic wall. The CNR at the 6 hours time point for unmodified micelles in WT mice or in ApoE-/- mice was not significantly different from pre-CNR, respectively $0.91 \pm 0.21$ and $1.26 \pm 0.35$. 
A group of ApoE-/- mice $(n=3)$ was also imaged at 30 minutes and 2 hours after the injection of unmodified micelles, time points which represent, respectively, $1 / 4$ and one half-life of the unmodified micelles. This control group was included as the blood clearance kinetics of the unmodified micelles was approximately twelve times faster than that of the tyrosine micelles and, therefore, in terms of half-lives, 30 minutes and 2 hours are the equivalent time points for the unmodified micelles to the 6 hour and 24 hour time points for the tyrosine micelles. This resulted in no apparent enhancement, with \% ENH values for the 30 minute and 2 hour time points of $5 \pm 20 \%$ and $1 \pm 19 \%$ respectively.

\section{Fluorescence microscopy}

To examine which components within plaque are preferentially bound by tyrosine grafted micelles, immunofluorescence staining was performed for various plaque components on aortic sections. To this aim, we first investigated whether tyrosine micelles were accumulating because of cellular uptake or by specific binding of components found in atherosclerotic plaques. At 6 hours, no uptake of the tyrosine micelles in the macrophages or macrophagederived foam cells was revealed (Figure 4). Staining for smooth-muscle cells with $\alpha$-actin did not evidence any micelle uptake (Figure 4). This suggests that tyrosine grafted micelles remained restricted to the extracellular space of the atherosclerotic plaque and that there was no cellular uptake of the contrast agent. Subsequently decorin, a matrix proteoglycan strongly expressed in atherosclerotic plaque, was investigated (30-32). Some co-localization was observed between the decorin and the distribution of tyrosine grafted micelles in the atherosclerotic plaque (Figure 5). Furthermore, we stained for collagen I, a major extracellular matrix component, but no co-localization was observed between collagen I and the tyrosine grafted micelles, since collagen I rich areas (green) did not co-localize with tyrosine micelle rich areas (Figure 5). Lastly, low-density lipoprotein, the major physical cholesterol transporter and a major component of the fatty streak found in the atherosclerotic plaque, was assessed with immunofluorescence. An association between ApoB and tyrosine micelles was clearly found by comparing the fluorescence from these two components in the plaque (Figure 5), which is a strong indication for the affinity of the tyrosine micelles for the lipoprotein-rich lipid cores in plaque.

\section{Discussion}

High spatial resolution, multisequential MRI allows the depiction of various components of atherothrombotic plaque, including fibrous tissue, lipids, calcium and thrombus formation (3,33-35). The development of atherosclerotic plaque is initiated by the deposition of lipid rich ApoB containing lipoproteins in susceptiblebut still pre-lesional areas of the arterial wall. The lipid deposition in the vessel wall occurs significantly before macrophage infiltration (2). Complications of atherosclerosis, such as plaque rupture, are the most common cause of death in the western world and are becoming a major health concern in developing countries (1). Previous reports have demonstrated a strong correlation between the lipid core size, thin fibrous cap and plaque instability (36-38). Binding the lipid core in the atherosclerotic plaque could help the diagnosis of plaque vulnerability. Such a contrast agent could also be used to identify plaque stabilization with lipid-lowering therapy (39-42).

We report here a tyrosine functionalized paramagnetic contrast agent that binds the lipid rich areas in the atherosclerotic plaque of ApoE-/- mice. Lipophilic properties, small particle size and prolonged circulation allow our contrast agent to penetrate and accumulate within the plaque after intravenous administration. As a result, tyrosine micelles efficiently highlight the atherosclerotic plaque in ApoE- $/-$ mice providing a high and specific enhancement in the plaque. In contrast, no or significantly less pronounced vessel wall enhancement was observed in the control groups. Simply targeting the micelles with an amino acid residue could represent 
a significant advance over targeting using more complex molecules such as antibodies, proteins or peptides, which have been applied previously (16-19).

The observed strong vessel wall MRI enhancement occurred at 6 hours which is significantly earlier than that found for similar contrast agents used in the past $(6,20,25,43)$. The evaluation of the distribution of tyrosine micelles revealed no cellular uptake. Tyrosine functionalized micelles are mainly observed within the extracellular matrix and co-localize well with ApoB, and slightly also with decorin. Proteoglycans like decorin have been shown to be critical in the retention of atherogenic lipoprotein inside the vessel wall $(30-32,44)$. These results strongly suggest that tyrosine micelles bind the lipid rich areas in atherosclerotic plaques.

Tyrosine micelles have a longer half-life compared to non-modified micelles (21 hours vs. 2 hours in ApoE-/- mouse model) which lead to more efficient vessel wall binding. The increase in the half-life of the tyrosine micelles suggest that they are transported by interactions with serum components. This carrier might also have a role in the uptake/exchange of tyrosine micelles in the atherosclerotic plaque and would allow local binding of tyrosine micelles. This could be explained by the binding of the tyrosine micelles to plasma lipoproteins, like HDL and/or LDL in the blood, which subsequently carry the paramagnetic micelles into the atherosclerotic plaque. Further experiments will be done to identify the serum component(s) responsible for this effect.

Binding the lipid rich areas in atherosclerotic plaque could help to discriminate the unstable plaque from stable plaque, as a large lipid core in combination with a thin fibrous cap strongly correlates with plaque instability $(4,45)$. Gadofluorine $\mathrm{M}$, a non-PEGylated amphiphilic micellar paramagnetic contrast agent has been shown in vitro and in vivo to target extracellular matrix proteins of atherosclerotic plaque without direct interaction of lipid rich areas $(5,6)$. Conversely, our novel tyrosine-based nanoparticles can effectively interact with lipid rich areas and thus could be used in conjunction with Gadofluorine $M$ to determine the ratio between lipid rich core and fibrous cap as a tool to detect plaque vulnerability.

Furthermore, the evaluation of the dynamic changes of the lipid content of the atherosclerotic plaque could represent a very valuable tool to assess the effect of lipid-lowering strategies, like statin therapy (39-42).

\section{Conclusion}

The combination of a high relaxivity, paramagnetic and versatile contrast agent that preferentially binds to lipophilic moieties led to an agent that can effectively detect lipid rich areas in the atherosclerotic plaque by MRI. Tyrosine micelles that bear gadolinium complexes may enable earlier detection of atherosclerotic plaques using MR imaging, since lipid deposition in the vessel wall occurs at very early stages of the development of atherosclerosis. In addition, the detection of lipid rich areas of the atherosclerotic plaque could allow the evaluation in other models of plaque vulnerability and the assessment of the effects of antiatherogenic therapies.

\section{Acknowledgments}

This study was supported in part by grant from the NIH/NHLBI R01 HL71021, NIH/NHLBI R01 HL078667 (ZAF), and also by ATHIM project, Médicen Ile-de-France.

\section{References}

1. Sanz J, Moreno PR, Fuster V. The year in atherothrombosis. J Am Coll Cardiol 2008;51(9):944-955. [PubMed: 18308164] 
2. Tabas I, Williams KJ, Boren J. Subendothelial lipoprotein retention as the initiating process in atherosclerosis: update and therapeutic implications. Circulation 2007;116(16):1832-1844. [PubMed: 17938300]

3. Honda M, Kawahara I, Kitagawa N, Tsutsumi K, Morikawa M, Hayashi T, Nagata I. Asymptomatic carotid artery plaques: use of magnetic resonance imaging to characterize vulnerable plaques in 6 cases. Surg Neurol 2007;67(1):35-39. [PubMed: 17210293]

4. Trivedi RA, UKing-Im JM, Graves MJ, Horsley J, Goddard M, Kirkpatrick PJ, Gillard JH. MRI-derived measurements of fibrous-cap and lipid-core thickness: the potential for identifying vulnerable carotid plaques in vivo. Neuroradiology 2004;46(9):738-743. [PubMed: 15309350]

5. Sirol M, Itskovich VV, Mani V, Aguinaldo JG, Fallon JT, Misselwitz B, Weinmann HJ, Fuster V, Toussaint JF, Fayad ZA. Lipid-rich atherosclerotic plaques detected by gadofluorine-enhanced in vivo magnetic resonance imaging. Circulation 2004;109(23):2890-2896. [PubMed: 15184290]

6. Meding J, Urich M, Licha K, Reinhardt M, Misselwitz B, Fayad ZA, Weinmann HJ. Magnetic resonance imaging of atherosclerosis by targeting extracellular matrix deposition with Gadofluorine M. Contrast Media Mol Imaging 2007;2(3):120-129. [PubMed: 17557276]

7. Aime, S.; Botta, M.; Terreno, E. Advances in Inorganic Chemistry. Vol. 57. Academic Press; 2005. Gd(III)-based contrast agents for MRI; p. 173-237.

8. Broz P, Marsch S, Hunziker P. Targeting of Vulnerable Plaque Macrophages with Polymer-Based Nanostructures. Trends Cardiovasc Med 2007;17(6):190-196. [PubMed: 17662913]

9. Lattuada L, Lux G. Synthesis of Gd-DTPA-cholesterol: a new lipophilic gadolinium complex as a potential MRI contrast agent. Tetrahedron Lett 2003;44(20):3893-3895.

10. Yu G, Yamashita M, Aoshima K, Takahashi M, Oshikawa T, Takayanagi H, Laurent S, Burtea C, Vander Elst L, Muller RN. A glycosylated complex of gadolinium, a new potential contrast agent for magnetic resonance angiography? Bioorg Med Chem Lett 2007;17(8):2246-2249. [PubMed: 17303417]

11. Lusis AJ. Atherosclerosis. Nature 2000;407(6801):233-241. [PubMed: 11001066]

12. Datta G, Epand RF, Epand RM, Chaddha M, Kirksey MA, Garber DW, Lund-Katz S, Phillips MC, Hama S, Navab M, Fogelman AM, Palgunachari MN, Segrest JP, Anantharamaiah GM. Aromatic residue position on the nonpolar face of class a amphipathic helical peptides determines biological activity. J Biol Chem 2004;279(25):26509-26517. [PubMed: 15075321]

13. Shah PK, Chyu KY. Apolipoprotein A-I mimetic peptides: potential role in atherosclerosis management. Trends Cardiovasc Med 2005;15(8):291-296. [PubMed: 16297766]

14. Anantharamaiah GM, Jones JL, Brouillette CG, Schmidt CF, Chung BH, Hughes TA, Bhown AS, Segrest JP. Studies of synthetic peptide analogs of the amphipathic helix. Structure of complexes with dimyristoyl phosphatidylcholine. J Biol Chem 1985;260(18):10248-10255. [PubMed: 4019510]

15. Navab M, Anantharamaiah GM, Reddy ST, Hama S, Hough G, Grijalva VR, Yu N, Ansell BJ, Datta G, Garber DW, Fogelman AM. Apolipoprotein A-I mimetic peptides. Arterioscler Thromb Vasc Biol 2005;25(7):1325-1331. [PubMed: 15831812]

16. Amirbekian V, Lipinski MJ, Briley-Saebo KC, Amirbekian S, Aguinaldo JG, Weinreb DB, Vucic E, Frias JC, Hyafil F, Mani V, Fisher EA, Fayad ZA. Detecting and assessing macrophages in vivo to evaluate atherosclerosis noninvasively using molecular MRI. Proc Natl Acad Sci U S A 2007;104 (3):961-966. [PubMed: 17215360]

17. van Tilborg GA, Mulder WJ, Deckers N, Storm G, Reutelingsperger CP, Strijkers GJ, Nicolay K. Annexin A5-functionalized bimodal lipid-based contrast agents for the detection of apoptosis. Bioconjug Chem 2006;17(3):741-749. [PubMed: 16704213]

18. Winter PM, Neubauer AM, Caruthers SD, Harris TD, Robertson JD, Williams TA, Schmieder AH, Hu G, Allen JS, Lacy EK, Zhang H, Wickline SA, Lanza GM. Endothelial alpha(v)beta3 integrintargeted fumagillin nanoparticles inhibit angiogenesis in atherosclerosis. Arterioscler Thromb Vasc Biol 2006;26(9):2103-2109. [PubMed: 16825592]

19. Zheng G, Chen J, Li H, Glickson JD. Rerouting lipoprotein nanoparticles to selected alternate receptors for the targeted delivery of cancer diagnostic and therapeutic agents. Proc Natl Acad Sci U S A 2005;102(49):17757-17762. [PubMed: 16306263] 
20. Mulder WJ, Strijkers GJ, Briley-Saboe KC, Frias JC, Aguinaldo JG, Vucic E, Amirbekian V, Tang C, Chin PT, Nicolay K, Fayad ZA. Molecular imaging of macrophages in atherosclerotic plaques using bimodal PEG-micelles. Magn Reson Med 2007;58(6):1164-1170. [PubMed: 18046703]

21. Torchilin VP. Structure and design of polymeric surfactant-based drug delivery systems. J Control Release 2001;73(2-3):137-172. [PubMed: 11516494]

22. Lukyanov AN, Torchilin VP. Micelles from lipid derivatives of water-soluble polymers as delivery systems for poorly soluble drugs. Adv Drug Deliv Rev 2004;56(9):1273-1289. [PubMed: 15109769]

23. Torchilin VP. Polymeric contrast agents for medical imaging. Curr Pharm Biotechnol 2000;1(2):183215. [PubMed: 11467336]

24. Jawien J, Nastalek P, Korbut R. Mouse models of experimental atherosclerosis. J Physiol Pharmacol 2004;55(3):503-517. [PubMed: 15381823]

25. Briley-Saebo KC, Amirbekian V, Mani V, Aguinaldo JG, Vucic E, Carpenter D, Amirbekian S, Fayad ZA. Gadolinium mixed-micelles: effect of the amphiphile on in vitro and in vivo efficacy in apolipoprotein E knockout mouse models of atherosclerosis. Magn Reson Med 2006;56(6):13361346. [PubMed: 17089381]

26. Van Butsele K, Sibret P, Fustin CA, Gohy JF, Passirani C, Benoit JP, Jerome R, Jerome C. Synthesis and $\mathrm{pH}$-dependent micellization of diblock copolymer mixtures. J Colloid Interface Sci 2009;329(2): 235-243. [PubMed: 18930246]

27. Briley-Saebo KC, Shaw PX, Mulder WJM, Choi S-H, Vucic E, Aguinaldo JGS, Witztum JL, Fuster V, Tsimikas S, Fayad ZA. Targeted Molecular Probes for Imaging Atherosclerotic Lesions With Magnetic Resonance Using Antibodies That Recognize Oxidation-Specific Epitopes. Circulation 2008;117(25):3206-3215. [PubMed: 18541740]

28. Nakashima Y, Plump AS, Raines EW, Breslow JL, Ross R. ApoE-deficient mice develop lesions of all phases of atherosclerosis throughout the arterial tree. Arterioscler Thromb 1994;14(1):133-140. [PubMed: 8274468]

29. Lukyanov AN, Gao Z, Torchilin VP. Micelles from polyethylene glycol/phosphatidylethanolamine conjugates for tumor drug delivery. J Control Release 2003;91(1-2):97-102. [PubMed: 12932641]

30. Rodriguez-Lee M, Bondjers G, Camejo G. Fatty acid-induced atherogenic changes in extracellular matrix proteoglycans. Curr Opin Lipidol 2007;18(5):546-553. [PubMed: 17885426]

31. Fischer JW, Steitz SA, Johnson PY, Burke A, Kolodgie F, Virmani R, Giachelli C, Wight TN. Decorin Promotes Aortic Smooth Muscle Cell Calcification and Colocalizes to Calcified Regions in Human Atherosclerotic Lesions. Arterioscler Thromb Vasc Biol 2004;24(12):2391-2396. [PubMed: 15472131]

32. Nakashima Y, Fujii H, Sumiyoshi S, Wight TN, Sueishi K. Early human atherosclerosis: accumulation of lipid and proteoglycans in intimal thickenings followed by macrophage infiltration. Arterioscler Thromb Vasc Biol 2007;27(5):1159-1165. [PubMed: 17303781]

33. Saam T, Ferguson MS, Yarnykh VL, Takaya N, Xu D, Polissar NL, Hatsukami TS, Yuan C. Quantitative evaluation of carotid plaque composition by in vivo MRI. Arterioscler Thromb Vasc Biol 2005;25(1):234-239. [PubMed: 15528475]

34. Yuan C, Mitsumori LM, Ferguson MS, Polissar NL, Echelard D, Ortiz G, Small R, Davies JW, Kerwin WS, Hatsukami TS. In vivo accuracy of multispectral magnetic resonance imaging for identifying lipid-rich necrotic cores and intraplaque hemorrhage in advanced human carotid plaques. Circulation 2001;104(17):2051-2056. [PubMed: 11673345]

35. Toussaint J-F, LaMuraglia GM, Southern JF, Fuster V, Kantor HL. Magnetic Resonance Images Lipid, Fibrous, Calcified, Hemorrhagic, and Thrombotic Components of Human Atherosclerosis In Vivo. Circulation 1996;94(5):932-938. [PubMed: 8790028]

36. Stary HC, Chandler AB, Dinsmore RE, Fuster V, Glagov S, Insull W Jr, Rosenfeld ME, Schwartz CJ, Wagner WD, Wissler RW. A Definition of Advanced Types of Atherosclerotic Lesions and a Histological Classification of Atherosclerosis : A Report From the Committee on Vascular Lesions of the Council on Arteriosclerosis, American Heart Association. Circulation 1995;92(5):1355-1374. [PubMed: 7648691]

37. Virmani R, Burke AP, Farb A, Kolodgie FD. Pathology of the Vulnerable Plaque. J Am Coll Cardiol 2006;47(8 Supplement 1):C13-C18. [PubMed: 16631505] 
38. Wasserman BA, Sharrett AR, Lai S, Gomes AS, Cushman M, Folsom AR, Bild DE, Kronmal RA, Sinha S, Bluemke DA. Risk factor associations with the presence of a lipid core in carotid plaque of asymptomatic individuals using high-resolution MRI: the multi-ethnic study of atherosclerosis (MESA). Stroke 2008;39(2):329-335. [PubMed: 18174475]

39. Lee JMS, Choudhury RP. Prospects for atherosclerosis regression through increase in high-density lipoprotein and other emerging therapeutic targets. Heart 2007;93(5):559-564. [PubMed: 16449520]

40. Lee JMS, Wiesmann F, Shirodaria C, Leeson P, Petersen SE, Francis JM, Jackson CE, Robson MD, Neubauer S, Channon KM, Choudhury RP. Early changes in arterial structure and function following statin initiation: Quantification by magnetic resonance imaging. Atherosclerosis 2008;197(2):951958. [PubMed: 17977546]

41. Zhao X-Q, Yuan C, Hatsukami TS, Frechette EH, Kang X-J, Maravilla KR, Brown BG. Effects of Prolonged Intensive Lipid-Lowering Therapy on the Characteristics of Carotid Atherosclerotic Plaques In Vivo by MRI: A Case-Control Study. Arterioscler Thromb Vasc Biol 2001;21(10):16231629. [PubMed: 11597936]

42. Corti R, Fayad ZA, Fuster V, Worthley SG, Helft G, Chesebro J, Mercuri M, Badimon JJ. Effects of lipid-lowering by simvastatin on human atherosclerotic lesions: a longitudinal study by highresolution, noninvasive magnetic resonance imaging. Circulation 2001;104(3):249-252. [PubMed: 11457739]

43. Frias JC, Ma Y, Williams KJ, Fayad ZA, Fisher EA. Properties of a versatile nanoparticle platform contrast agent to image and characterize atherosclerotic plaques by magnetic resonance imaging. Nano Lett 2006;6(10):2220-2224. [PubMed: 17034087]

44. Skalen K, Gustafsson M, Rydberg EK, Hulten LM, Wiklund O, Innerarity TL, Boren J. Subendothelial retention of atherogenic lipoproteins in early atherosclerosis. Nature 2002;417(6890):750-754. [PubMed: 12066187]

45. Maseri A, Fuster V. Is there a vulnerable plaque? Circulation 2003;107(16):2068-2071. [PubMed: 12719286] 


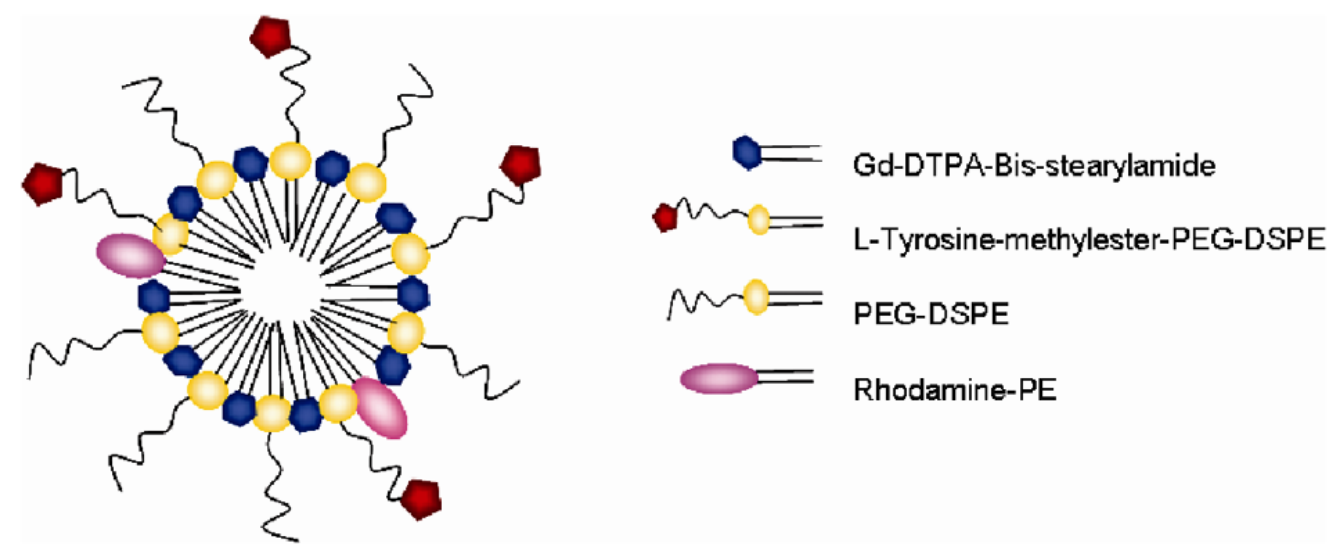

Figure 1.

Tyrosine micelle schematic diagram. The micelle composition (molar ratio) is the following: Gd-DTPA-Bis-stearylamide 50\%, L-Tyrosine-methylester-PEG 2000 -DSPE $15 \%, \mathrm{PEG}_{2000^{-}}$ DSPE $35 \%$ and Rhodamine-PE $0.01 \%$. 


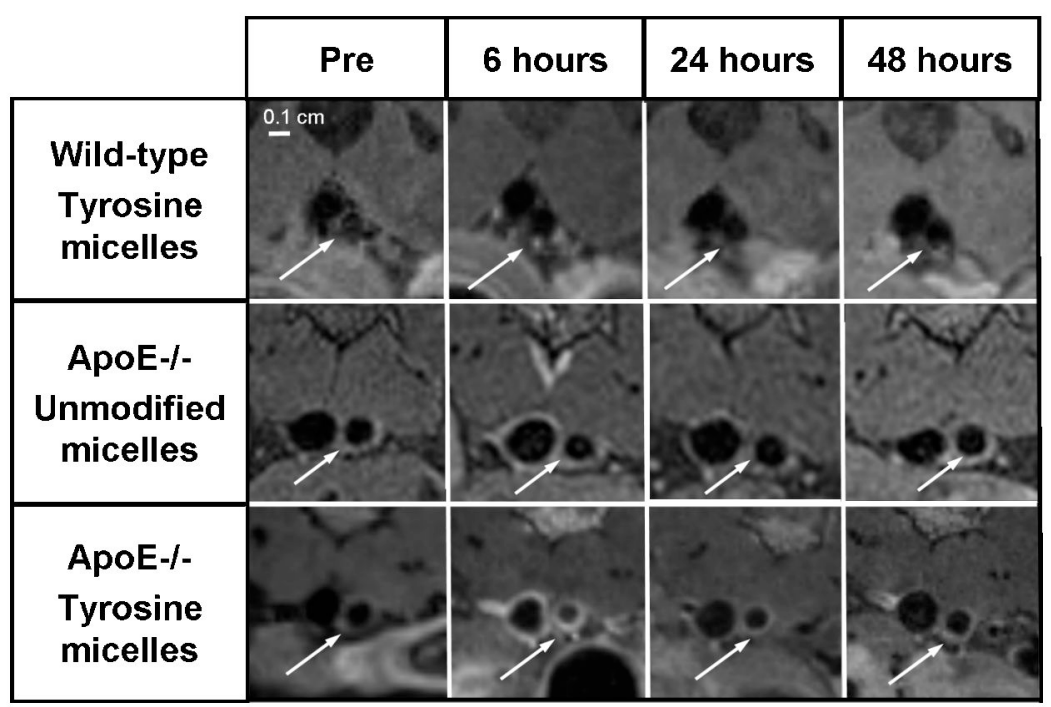

Figure 2. Tyrosine PEG-micelles uptake in ApoE-/-, wild-type mice and unmodified PEG-micelles uptake in ApoE-/- mice

Representative magnified in vivo magnetic resonance images of the abdominal aorta in wildtype mouse injected with tyrosine PEG-micelles (top). ApoE-/- mouse injected with unmodified PEG-micelles (middle) or with tyrosine PEG-micelles (bottom) imaged at different time points. Images were obtained at $9.4 \mathrm{~T}$ using a T1-weighted black-blood sequence. White arrows indicate the abdominal aorta. 


\section{* Wall enhancement}

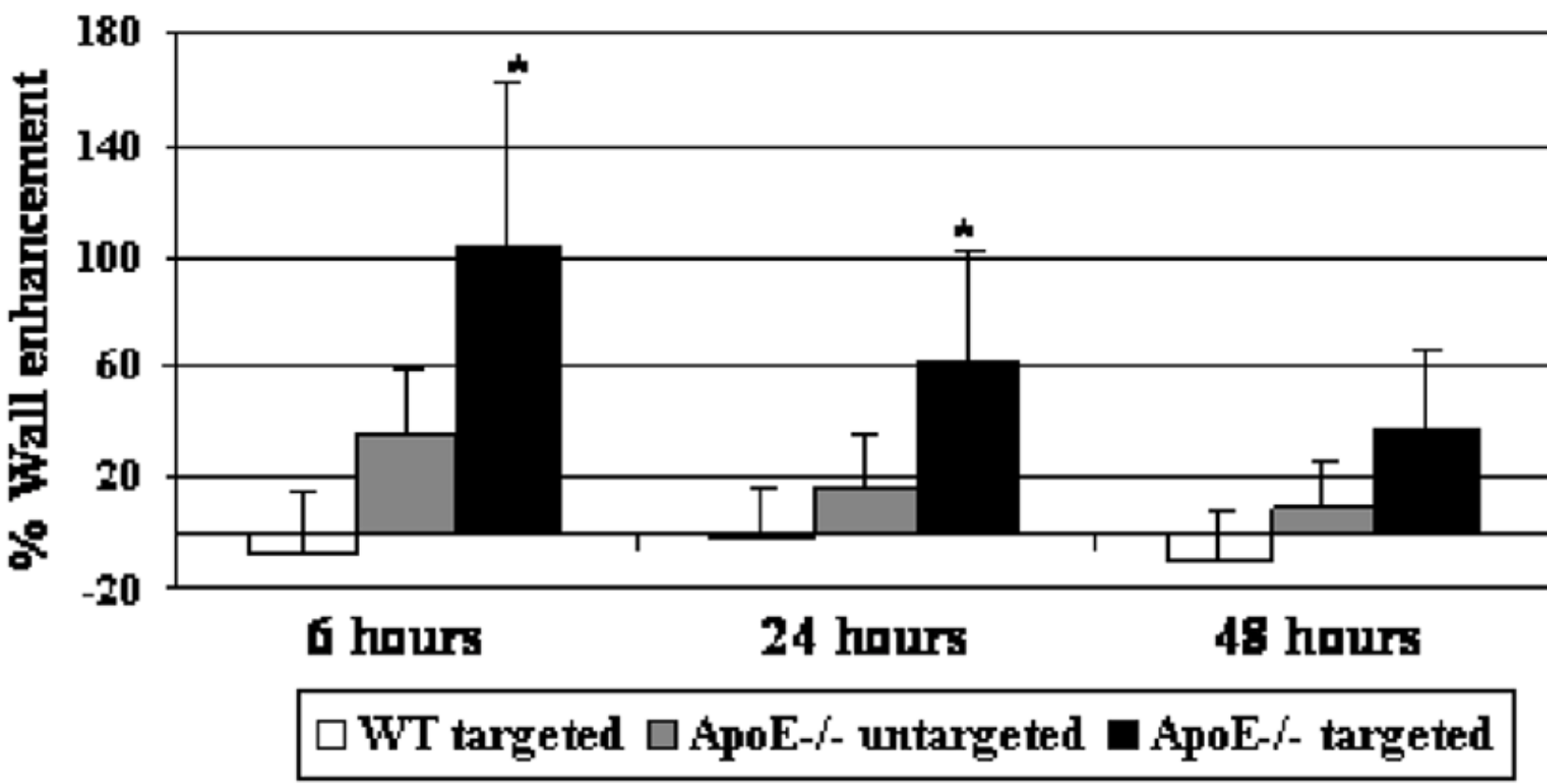

Figure 3. Quantification of the wall enhancement in ApoE-/- and in WT mice

Enhancement at 6, 24 and 48 hours post-injection of wall aorta in WT mice injected with tyrosine micelles and in ApoE-/- mice injected with unmodified or micelles. Unmodified micelles and tyrosine micelles were injected at the same Gd concentration $60 \mu \mathrm{mol} / \mathrm{kg}$. $(* \mathrm{p}<0.05)$ 


\section{$\alpha$-actin}

\section{Macrophages}

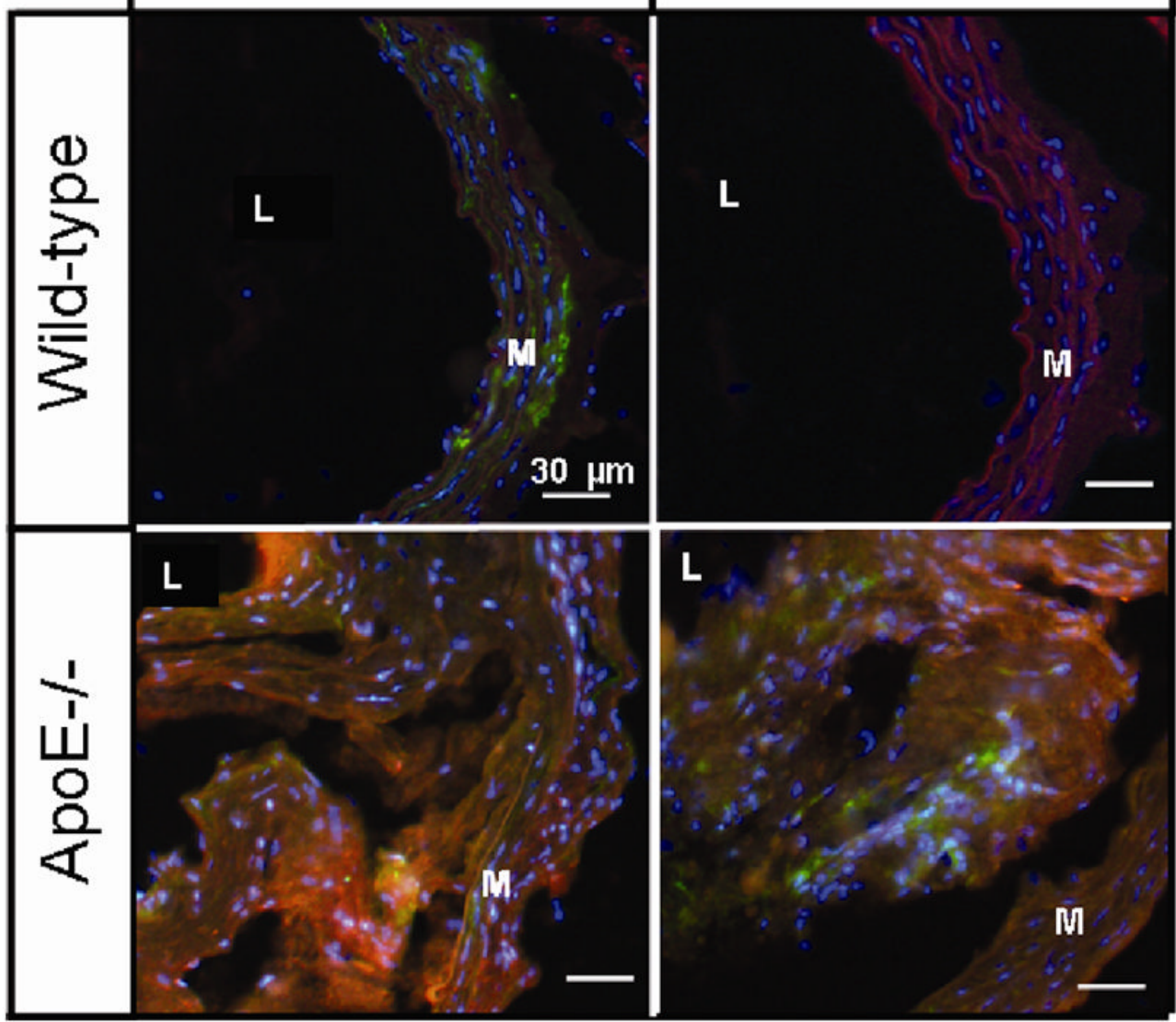

Figure 4. Lack of cellular co-localization of tyrosine PEG-micelle in ApoE-I- mice Localization of tyrosine grafted micelles and of ApoE-/- and WT mice. Fluorescence microscopy at 6 hours pi of tyrosine grafted micelles (red) with staining of various cells type; $\alpha$-actin for smooth muscle cell and CD68 for macrophages(green). Nuclei were stained with DAPI. The media and lumen are labeled M and L, respectively. 


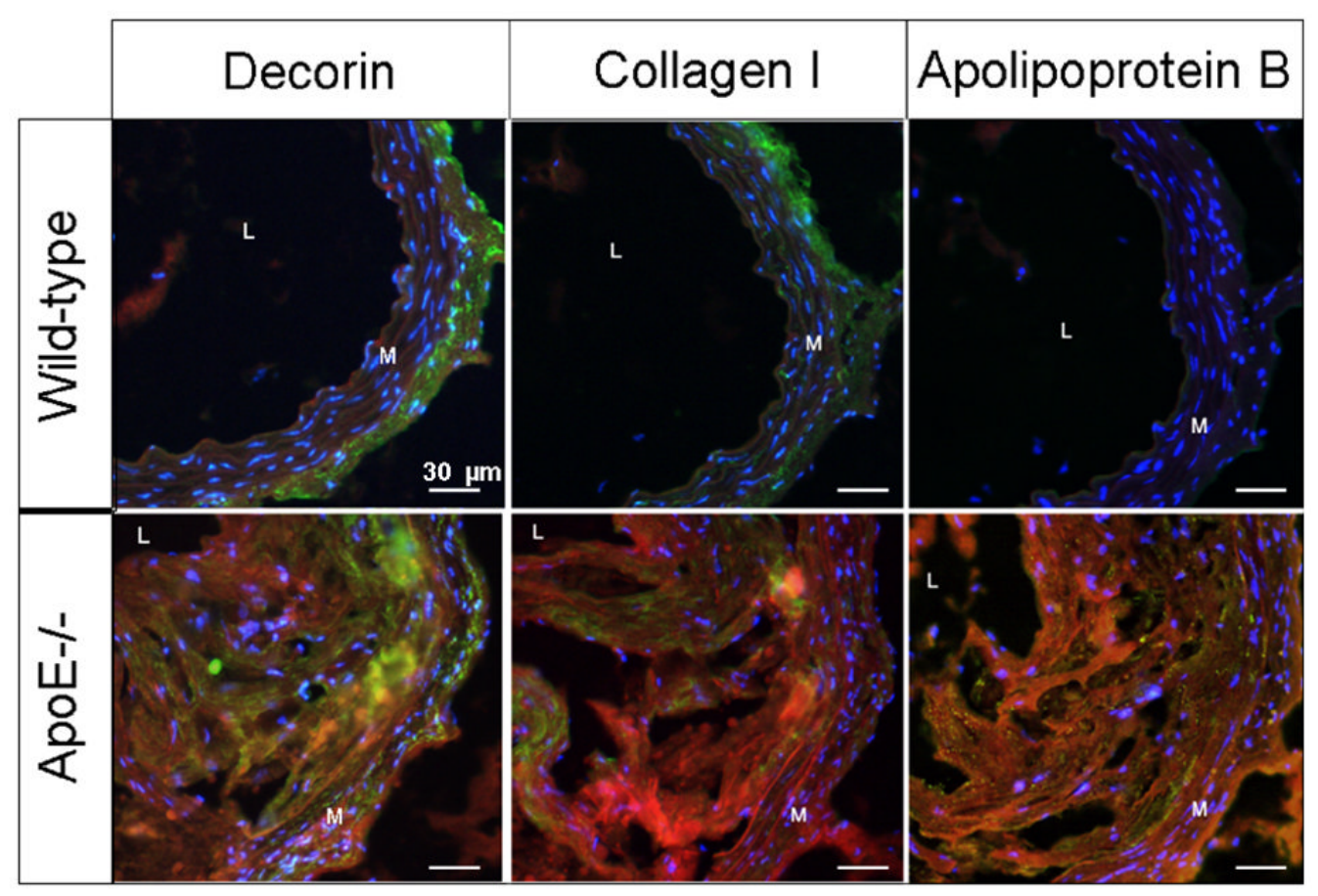

Figure 5. Fluorescence microscopy

Localization of tyrosine grafted micelles in the extracellular matrix of ApoE-/- and WT mice. Fluorescence microscopy at 6 hours pi of tyrosine grafted micelles (red) with staining of various extracellular matrix components; decorin, collagen I and ApoB (green). Nuclei were stained with DAPI. The media and lumen are labeled $\mathrm{M}$ and L, respectively. 


\section{Table 1}

Physical properties of tyrosine micelles and unmodified micelles

\begin{tabular}{lcccc}
\hline & [Tyrosine] (\% PEG-chains) & $\begin{array}{c}\text { Particule size (nm) } \\
\text { (z-average } \pm \text { disp.) }\end{array}$ & $\mathbf{r 1 ~}^{\left(\mathbf{m M}^{\mathbf{- 1}} \mathbf{~ s}^{\mathbf{- 1}}\right)}$ & $\begin{array}{c}\text { Half-life in } \\
\text { ApoE-l- } \\
\text { (hours) }\end{array}$ \\
\hline Unmodified micelles & 0 & $12 \pm 2.7$ & $10.1 \pm 1.5$ & 2 \\
Tyrosine micelles & $15 \% \pm 4$ & $14.6 \pm 2.5$ & $10.3 \pm 0.9$ & 21 \\
\hline
\end{tabular}

